\title{
An optimized QFP structure for use in radio frequency multi-chip module applications
}

\author{
Haiyan SUN ${ }^{1,2 a)}$, Zhikuang CAI ${ }^{1}$, Jianhui WU ${ }^{1}$, \\ and Longxing $\mathbf{S H I}^{1}$ \\ ${ }^{1}$ National ASIC System Engineering Research Center, Southeast University, \\ Nanjing 210096, People's Republic of China \\ 2 Jiangsu Provincial Key Lab of ASIC Design, Nantong University, \\ Nantong 226019, People's Republic of China \\ a)sun.yan@seu.edu.cn
}

\begin{abstract}
This paper presents the design and demonstration of an optimized quad flat pack (QFP) structure for radio frequency (RF) multi-chip module (MCM) application. In order to reduce large impedance discontinuities in the low-cost QFP, a new design scheme with cascade coplanar transmission line structure built into the lead frame has been developed. The optimized structure is accurately modeled in 3D model by utilizing ANSYS HFSS. S-parameter is utilized to help in understanding the contributing to the optimized QFP structure. The analysis results indicate that the optimized QFP structure can be fully capable of supporting $5.8 \mathrm{GHz}$ RF MCM application.
\end{abstract}

Keywords: multi-chip module (MCM), quad flat package (QFP), electromagnetic modeling, coplanar transmission line structure

Classification: Integrated circuits

\section{References}

[1] J.-H. Kim, R. Schmitt, D. Oh, W. Beyene, M. Li, A. Vaidyanath, J. Feng, and C. Yuan, "Feasibility Study of a $3.2 \mathrm{~Gb} / \mathrm{s}$ Memory Interface in Ultra Low-Cost LQFP Packages," DesignCon 2009, Santa Clara, USA, Feb. 2009.

[2] R. Oikawa, "A Low-Cost QFP Design for over 8 Gbps SerDes Applications with Inductive Return Path Co-Planar Waveguide Built into the Lead Frame," Proc. 60th Electronic Components and Technology Conference, Las Vegas, USA, pp. 1479-1484, June 2010.

[3] U. Paoletti, T. Hisakado, and O. Wada, "Quasi-Static Lumped Element Stand-Alone Package Model for Quad Flat Package," Proc. Electrical Design of Advanced Packaging and Systems Symposium, pp. 57-60, Dec. 2008.

[4] D. Jessie and L. Larson, "Conformal mapping for buried CPW with finite grounds," Electronics Letters, vol. 37, no. 25, pp. 1521-1523, Dec. 2001. 


\section{Introduction}

Due to the ever-increasing demand for multifunctional, cheaper, smaller and higher performance consumer electronics, MCM has become an important technology to be used for the design of present and next generation microelectronic products. The main reason is that MCM could enable the integration of heterogeneous functions (e.g., high-speed processors, RF devices, sensors, etc.) in a single compact package module. QFP has been used for many years to house large scale or very large scale integrated circuits. Compared with ball grid array package (BGA), QFP can provide a low cost and doable solution to achieve MCM modules. However, at high operating frequencies in the $\mathrm{GHz}$ region, it becomes a challenge to properly design MCM modules to transmit broadband signals without degrading system performance, especially on using QFP technology [1,2]. One of the major problems when QFP used for RF MCM applications is impedance discontinuity. Parasitics of leads and bonding wires can significantly degrade the signal integrity at higher frequency.

Fig. 1 (a) illustrates the outline of a selected traditional QFP80 lead frame for MCM application. Two dies are bonded to the paddle. Bond wires are used to connect the pads with the leads. In order to illustrate the parasitic effects of the QFP80 package, commercial electromagnetic analysis software HFSS is used to simulate the Z parameter of signal path S1, as shown in Fig. 1 (a). Fig. 1 (b) shows the simulated result when $\mathrm{S} 1$ is shorted to ground. The Magnitude of Z11 rises dramatically due to the internal inductive reactances and exhibits a very strong resonance at $3.8 \mathrm{GHz}$. Clearly, QFP80 package is very difficult for circuits to work at these frequencies. Despite the concerns of high frequency loss, we believe that the signal transmission bandwidth of QFP80 can be increased for RF MCM applications by further optimized design while maintaining low cost.

In this paper, an optimized QFP80 package with cascade coplanar transmission line structure built into the lead frame has been developed to solve impedance discontinuities issue. Due to the complexity level in QFP struc-
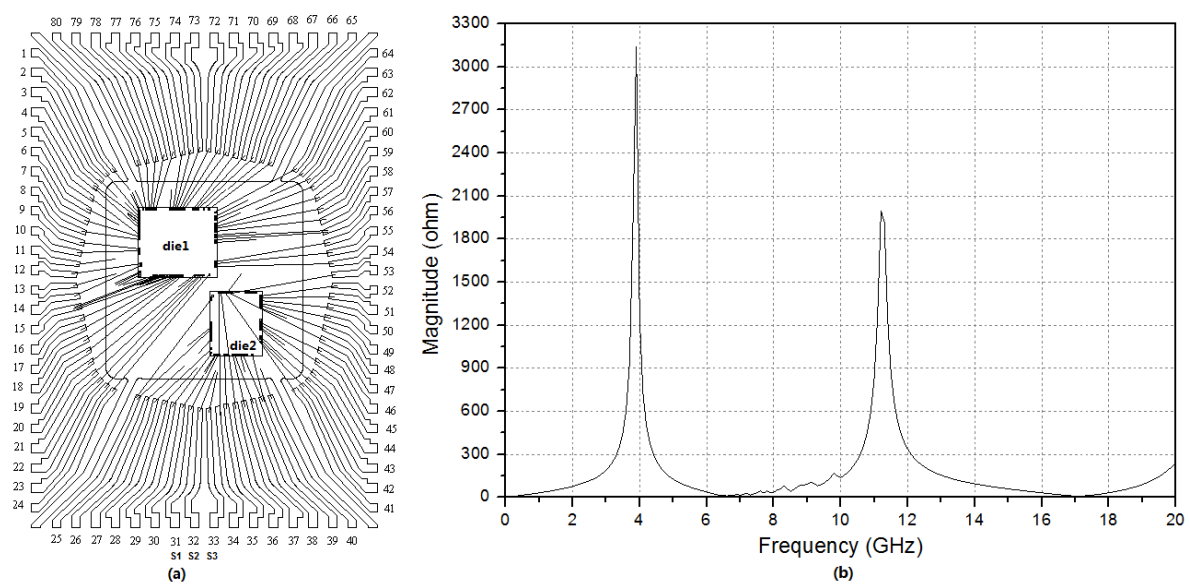

Fig. 1. (a) MCM package based on a traditional QFP80 package, (b) simulated result of Z11. 
ture, each interconnect segment is accurately modeled in 3D model by HFSS. S-parameter is used to help in understanding the contributing to the optimized QFP80 structure. Both the simulation and measurement are performed and compared to validate the design scheme.

\section{Construction of the coplanar transmission line structure}

In our work, we believe that if the QFP80 lead frame defined by characteristic impedance and electrical length may provide a better broadband transmission than traditional lumped elements mutually coupled to neighboring traces [3], as shown in Fig. 2. Compared with Fig. 2 (a), Fig. 2 (b) shows the proposed transmission line equivalent circuit model with the QFP80 lead frame designed as a coplanar transmission line structure. The raw coupling capacitance has been eliminated by tight coupling of the electric fields to the transmission line medium. Furthermore, the printed circuit board (PCB) transmission line can be effectively extended to the bond wire location. So, the purpose of optimal design is to make the discontinuity impedance of QFP80 lead frame to be close to the characteristic impedance of the PCB transmission line.

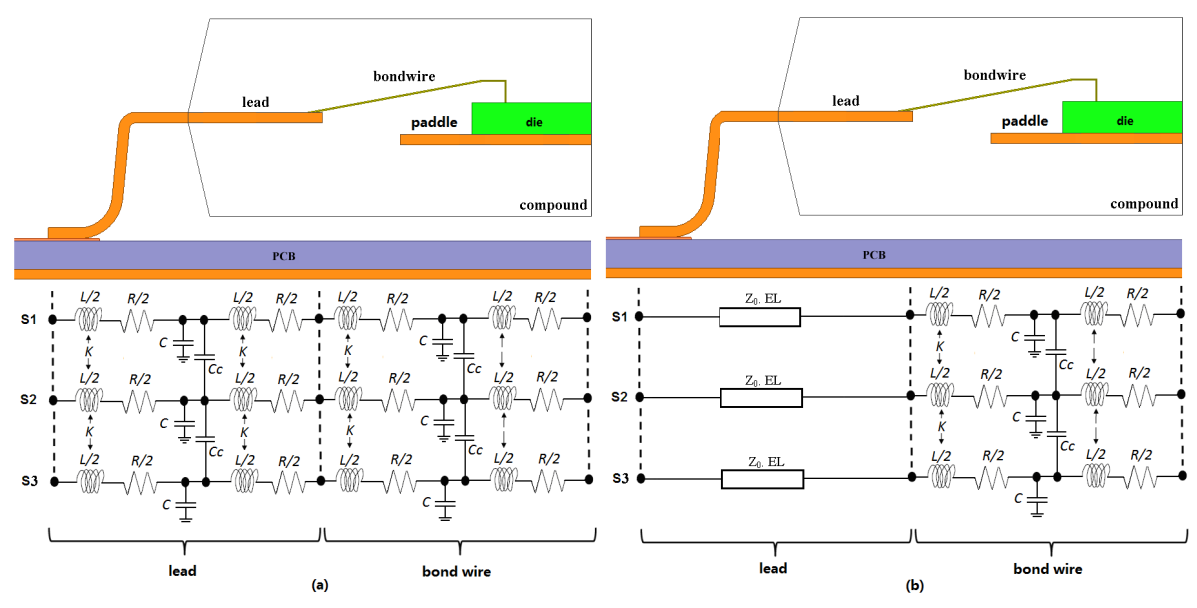

Fig. 2. (a) Simplified RLCM equivalent circuit model of QFP80, (b) transmission line equivalent circuit model of QFP80.

Fig. 3 illustrates the two cross-sectional views of the traditional structure (denoted by G-S-G) and proposed coplanar transmission line structure with finite irregular ground structure, respectively. Compared with Fig. 3 (a), two physical changes transform the QFP80 lead frame into coplanar transmission line structure, as shown in Fig. 3(b). The first is that the grounded leads S1 and S3 have been extended to connect the paddle and the signal lead S2 has been extended to close the paddle. This change not only can provide a better ground shield to control crosstalk, but also can short the bond wire to achieve a lower inductance. The second is to set the width of signal lead and the space between leads to constant value. This change can be used to provide impedance control for signal trace. It should be noticed that the 
outside part of the signal lead is embedded in air $\left(\varepsilon_{r} \approx 1\right)$, while the inside part is embedded in compound $\left(\varepsilon_{r}=3.3\right)$. Therefore, the full lead should be designed as a cascade coplanar structure.
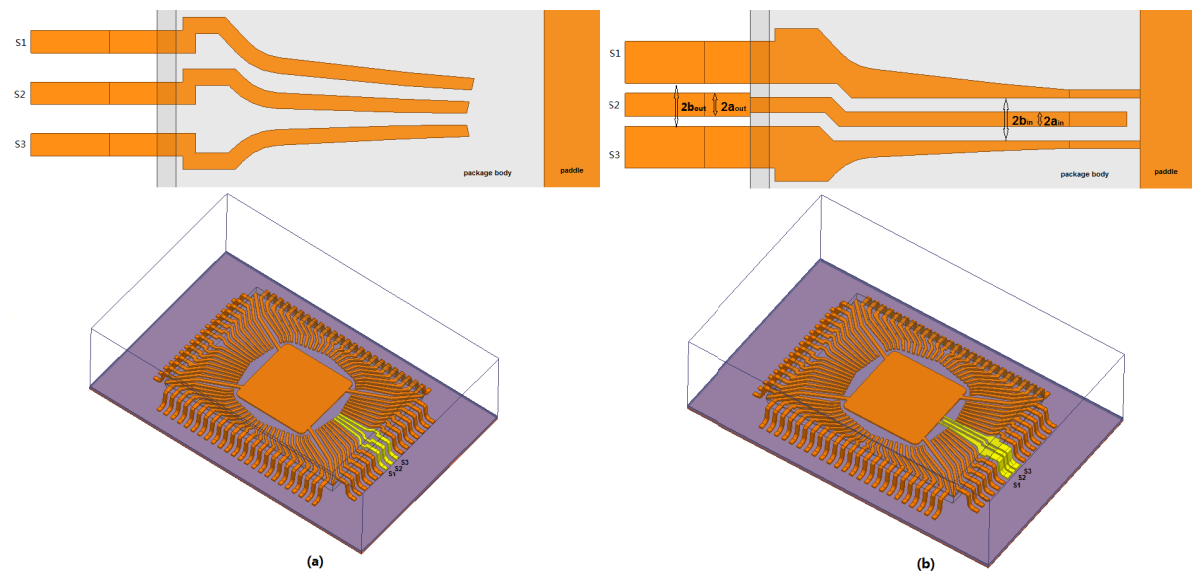

Fig. 3. Two cross-sectional views: (a) traditional structure (denoted by G-S-G) and (b) proposed coplanar topology with finite irregular ground structure.

Quasi-static analysis can be adopted to extract the transmission line capacitances, which can be used to derive the effective dielectric constant $\varepsilon_{e}$ and characteristic impedance $Z_{0}$ [4]. However, the frequency dependent parameters for this cascade coplanar structure are hard to quantify, and the discontinuity between the different segments is very complex especially at high frequency. So, ANSYS 3-D electromagnetic field solver HFSS, using finite element analysis, is adopted to simulate and analyze this structure. Six simulation configurations with different spacing and width are conducted to find a better compromise between performance, robustness and ease of fabrication, as shown in Table I. Fig. 4 shows S-parameters from $10 \mathrm{MHz}$ to $20 \mathrm{GHz}$ for these configurations. From Fig. 4 (a) and (b), the scenario 2 shows better performance over the whole $20 \mathrm{GHz}$ bandwidth. Furthermore, from Fig. 4 (c) and (d), the scenario 6 with shortest distance between the signal lead and grounded lead is best for impedance matching. But the $0.130 \mathrm{~mm}$ spacing

Table I. Simulation configurations.

\begin{tabular}{lllll}
\hline & \multicolumn{2}{c}{ inside part } & \multicolumn{2}{c}{ outside part } \\
\cline { 2 - 5 } Configuration & $2 a_{\text {in }}$ & $b_{\text {in }}-a_{\text {in }}$ & $2 a_{\text {out }}$ & $b_{\text {out }}-a_{\text {out }}$ \\
\hline Scenario 1 & $0.200 \mathrm{~mm}$ & $0.230 \mathrm{~mm}$ & & \\
Scenario 2 & $0.250 \mathrm{~mm}$ & $0.205 \mathrm{~mm}$ & & \\
Scenario 3 & $0.300 \mathrm{~mm}$ & $0.180 \mathrm{~mm}$ & & \\
Scenario 4 & & & $0.300 \mathrm{~mm}$ & $0.180 \mathrm{~mm}$ \\
Scenario 5 & & & $0.350 \mathrm{~mm}$ & $0.155 \mathrm{~mm}$ \\
Scenario 6 & & & $0.400 \mathrm{~mm}$ & $0.130 \mathrm{~mm}$ \\
\hline
\end{tabular}



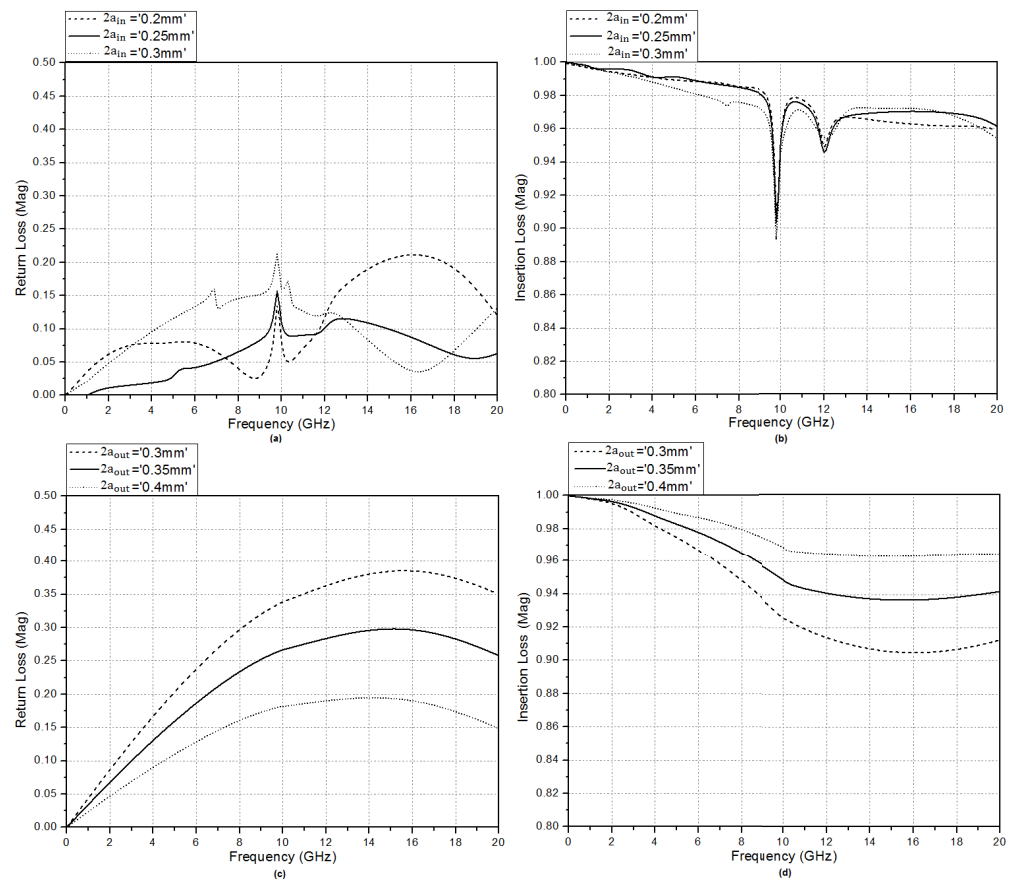

Fig. 4. S-parameter sweep results: (a) S11 and (b) S21 of inside part, (c) S11 and (d) S21 of outside part.
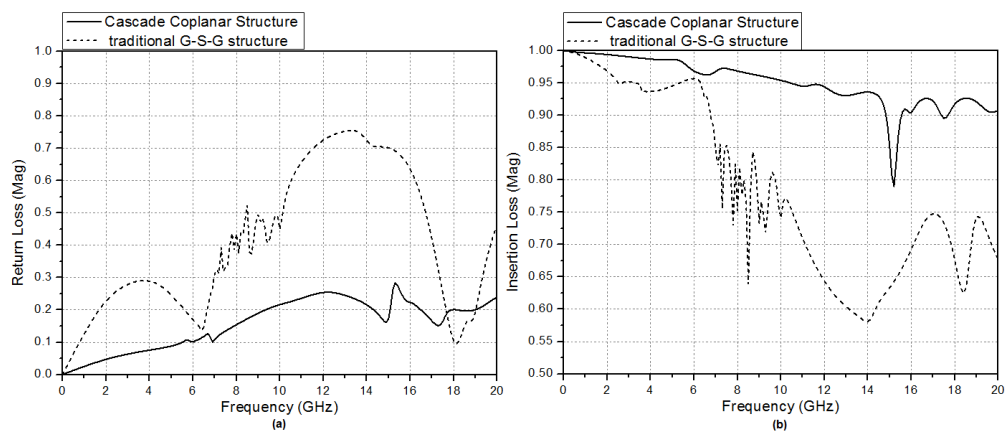

Fig. 5. Simulated (a) S11 and (b) S21 of the proposed cascade structure and traditional G-S-G structure.

is difficult to be fabricated. So, the proposed cascade structure can be constructed by combining scenario 2 and scenario 5. Fig. 5 shows the extensive simulation results of the full cascade coplanar transmission line structure and the traditional structure (denoted by G-S-G), as shown in Fig. 3. As we can see, the full cascade coplanar transmission line structure built into the lead frame is remarkable for discontinuity cancellation. This means that it is feasible to increase the bandwidth only by slight changes to the QFP80 lead frame before relying on expensive packaging solutions.

\section{RF MCM design based on the optimized QFP80 structure}

In this section, a full optimized QFP80 lead frame is designed and fabricated for $5.8 \mathrm{GHz}$ RF MCM package, as shown in Fig. 6. Fig. 6 (a) and (b) demonstrate the 2D design view and photograph of this optimized QFP80 lead frame, respectively. There are four high-frequency channels in this MCM 

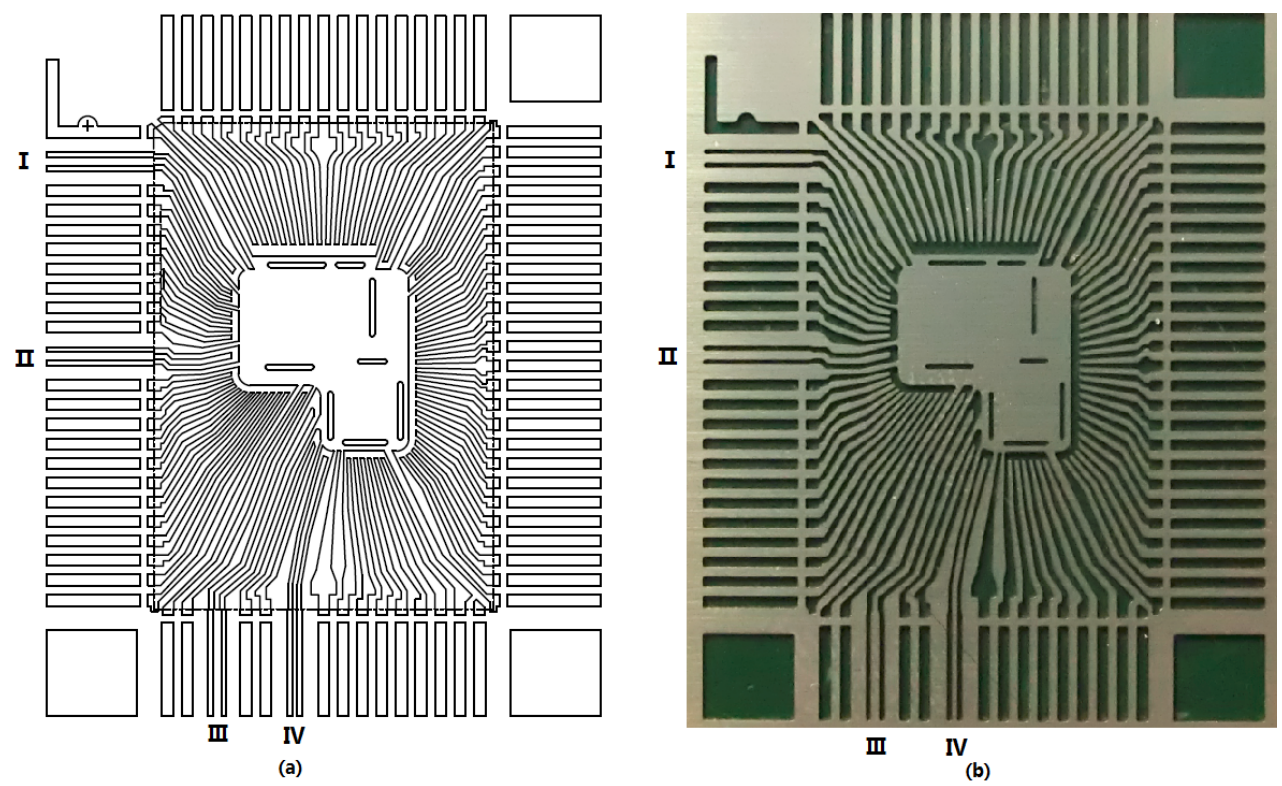

Fig. 6. (a) Two-dimensional design view and (b) photograph of the optimized QFP lead frame for RF MCM package.

package (denoted by I, II, III and IV). The channel I and II are designed for $5.8 \mathrm{GHz}$ RF signal transmission, and the channel III and IV are designed for $2.4 \mathrm{GHz} \mathrm{RF}$ signal transmission. According to the analysis results of section 2, the simulation configurations of the four channels are summarized in Table II. It should be noted that the simulation configuration of channel IV is different from others due to the compromise between the performance and ease of fabrication. Each signal port is also terminated with $50 \Omega$ load. The simulated results of these four channels are shown in Fig. 7. In order to evaluate the applicable frequency easily, the magnitude of $0.178(-15 \mathrm{~dB})$ is considered as criterion for bandwidth associated with return loss, and the magnitude of $0.891(-1 \mathrm{~dB})$ is considered as criterion for bandwidth associated with insertion loss. From Fig. 7 (a), the bandwidth of channel I, II, III and IV measured for $\mathrm{S} 11$ is $8 \mathrm{GHz}, 9.4 \mathrm{GHz}, 7.3 \mathrm{GHz}$ and $5 \mathrm{GHz}$, respectively. From Fig. 7 (b), the bandwidth of channel I, II, III and IV measured for S21 is $11.6 \mathrm{GHz}, 15.2 \mathrm{GHz}, 14.7 \mathrm{GHz}$ and $7.4 \mathrm{GHz}$, respectively. As we can see, the simulated results of channel I, II and III show good correlations with S11 and S21 of the proposed cascade coplanar transmission line structure in

Table II. Simulation configurations.

\begin{tabular}{lllll}
\hline & \multicolumn{2}{c}{ inside part } & \multicolumn{2}{c}{ outside part } \\
\cline { 2 - 5 } channel & $2 a_{\text {in }}$ & $b_{\text {in }}-a_{\text {in }}$ & $2 a_{\text {out }}$ & $b_{\text {out }}-a_{\text {out }}$ \\
\hline I & $0.250 \mathrm{~mm}$ & $0.205 \mathrm{~mm}$ & $0.350 \mathrm{~mm}$ & $0.155 \mathrm{~mm}$ \\
II & $0.250 \mathrm{~mm}$ & $0.205 \mathrm{~mm}$ & $0.350 \mathrm{~mm}$ & $0.155 \mathrm{~mm}$ \\
III & $0.250 \mathrm{~mm}$ & $0.205 \mathrm{~mm}$ & $0.350 \mathrm{~mm}$ & $0.155 \mathrm{~mm}$ \\
IV & $0.200 \mathrm{~mm}$ & $0.160 \mathrm{~mm}$ & $0.200 \mathrm{~mm}$ & $0.210 \mathrm{~mm}$ \\
\hline
\end{tabular}



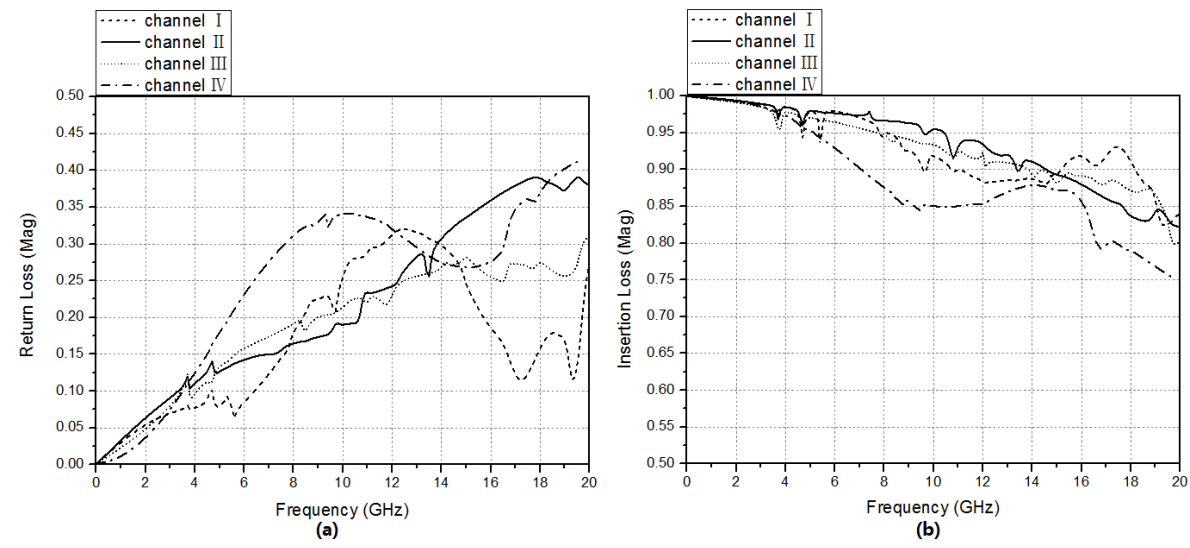

Fig. 7. Simulated return loss (a) and insertion loss (b) of the four channels in the optimized QFP lead frame.

Fig. 5. It should be noted that the performance of channel IV is degraded due to the different simulation configuration, but the bandwidth of it is adequate for $2.4 \mathrm{GHz}$ RF signal transmission. From above analysis, it is obvious that the cascade coplanar transmission line structure built in the lead frame can enhance the package bandwidth and it is suitable to be built in any place in QFP80 lead frame.

Fig. 8 (a) demonstrates the new 3D package model for $5.8 \mathrm{GHz}$ RF MCM
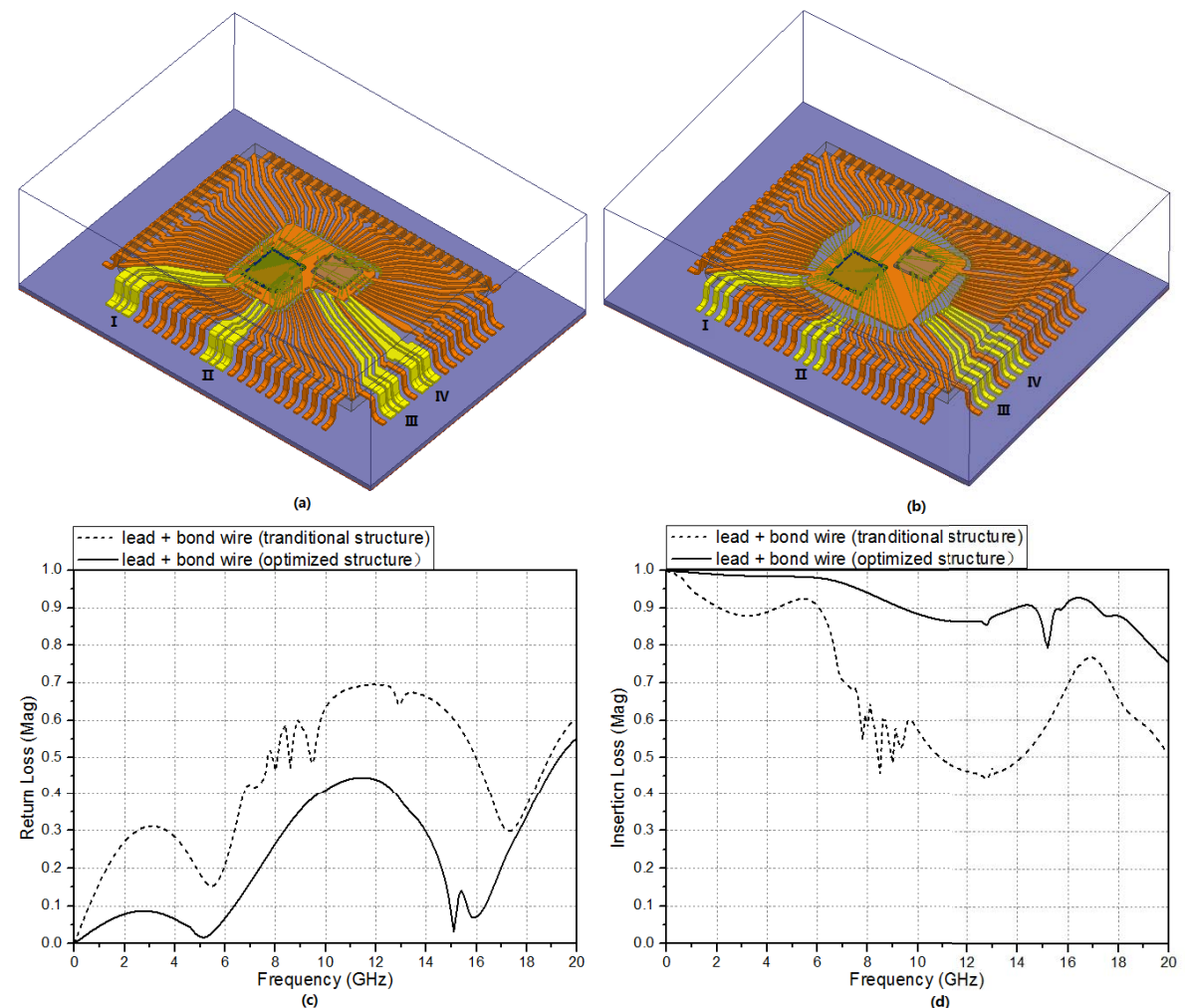

Fig. 8. (a) 3D view of the optimized MCM package, (b) $3 \mathrm{D}$ view of the traditional MCM package, (c) simulated return loss and (d) insertion loss of these two packages. (c) IEICE 2012

DOI: $10.1587 /$ elex.9.1666

Received October 09, 2012

Accepted October 19, 2012

Published November 09, 2012 
design based on the optimized QFP80 lead frame. At the same time, the $5.8 \mathrm{GHz}$ RF MCM package is modeled base on the traditional QFP80 lead frame for comparison, as shown in Fig. 8 (b). In order to show the performance of the optimized MCM, the selected channel I including leads and bond wires in both models is simulated in HFSS, respectively. It should be noted that the selected channel I is designed as the cascade coplanar transmission line structure in Fig. 8 (a), while in Fig. 8 (b), the selected channel I is modeled as traditional G-S-G structure. The simulated return loss (S11) and insertion loss (S21) of these two packages are shown in Fig. 8(c) and (d). Due to the influence of the inductive bond wires, the bandwidth of the optimized QFP package measured for S11 and S21 is decreased to $7.2 \mathrm{GHz}$
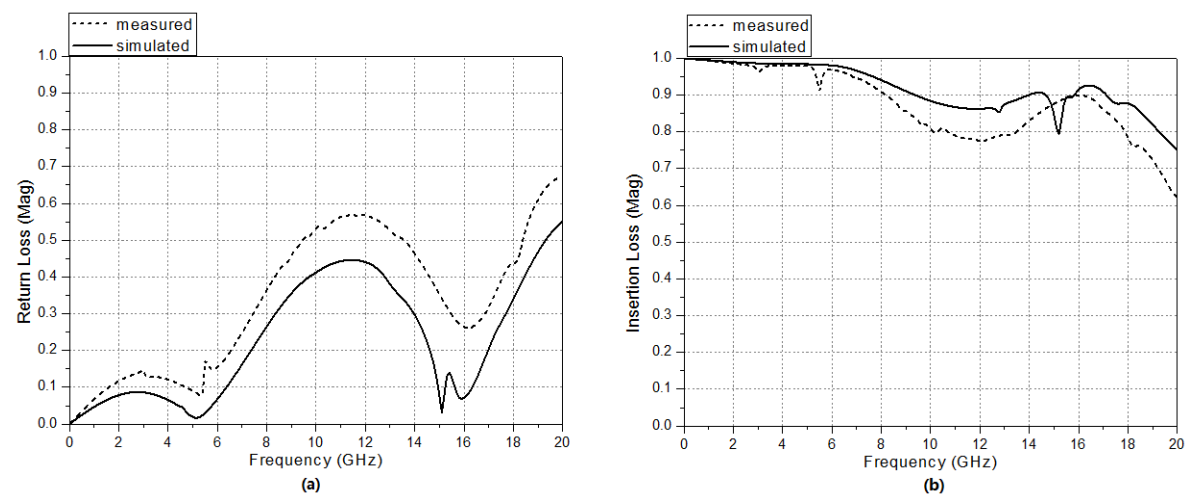

Fig. 9. The measured and simulated (a) return loss and (b) insertion loss versus frequency.

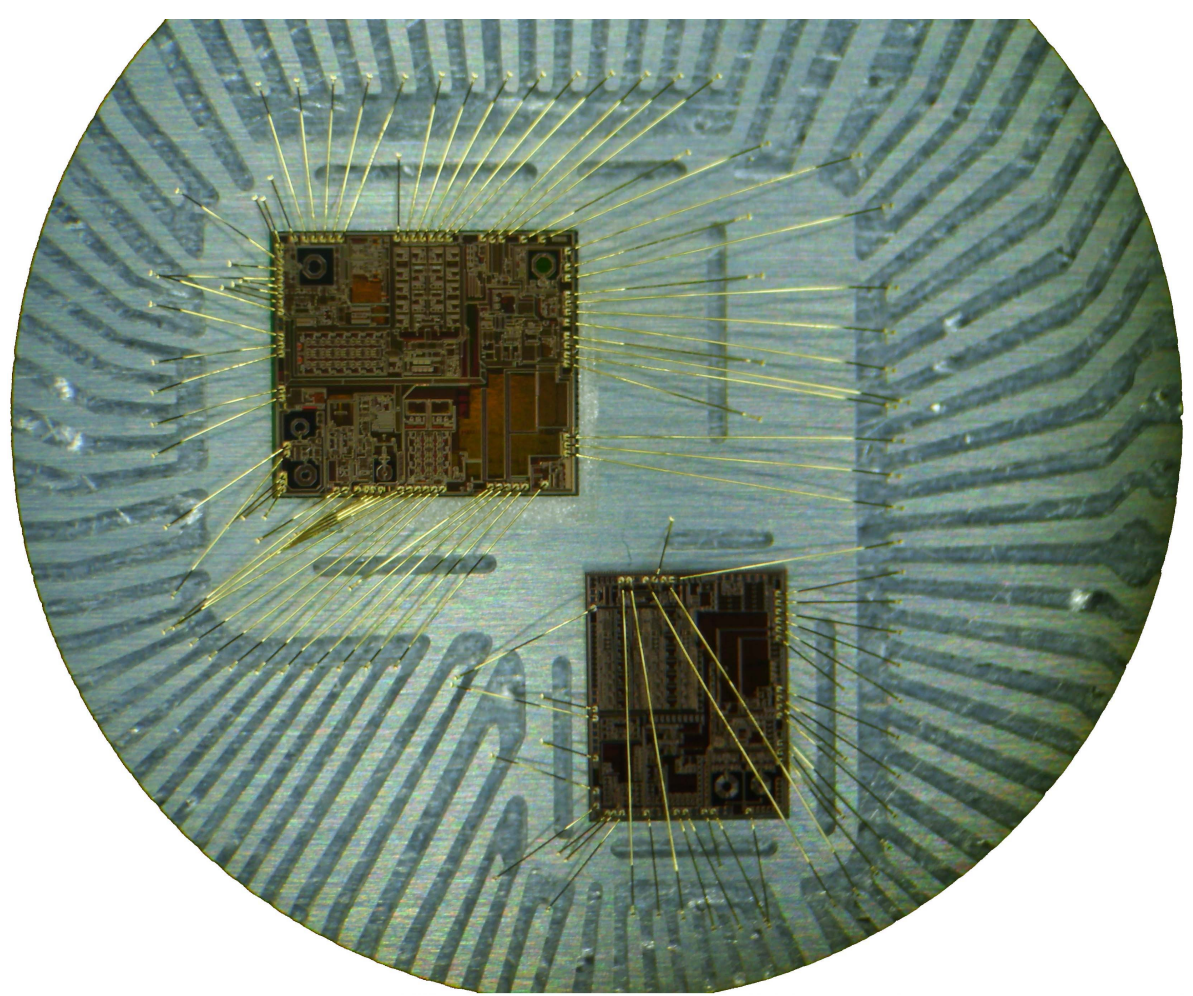

Fig. 10. The photograph of the new MCM based on the optimized QFP80 lead frame. 
(at $0.178 \mathrm{mag}$ ) and $9.5 \mathrm{GHz}$ (at $0.891 \mathrm{mag}$ ), while the bandwidth of the traditional QFP package measured for S11 and $\mathrm{S} 21$ is $1.2 \mathrm{GHz}$ (at $0.178 \mathrm{mag}$ ) and $2.4 \mathrm{GHz}$ (at $0.891 \mathrm{mag}$ ). Compared with the traditional G-S-G package, the simulated results indicate the feasibility of our optimized design. Furthermore, Fig. 9 shows the simulated results against measured data of the selected channel I. It can be seen that the return loss and insertion loss follow the same trends and show good agreements over the whole $20 \mathrm{GHz}$ bandwidth between the simulation and measurement. Due to the package processing error, the bandwidth measured for S11 and $\mathrm{S} 21$ is $6.3 \mathrm{GHz}$ (at $0.178 \mathrm{mag}$ ) and $8.3 \mathrm{GHz}$ (at $0.891 \mathrm{mag}$ ) from measured data, respectively. The results indicate that the optimized QFP80 package can be fully capable of supporting $5.8 \mathrm{GHz}$ RF MCM design. The photograph of the new MCM package based on the optimized QFP80 lead frame is demonstrated in Fig. 10.

\section{Conclusion}

In this paper, a full optimized QFP80 package with four cascade coplanar transmission line structures built in has been successfully developed for 5.8 GHz RF MCM application. Additionally, this design scheme could be readily applied not only to the QFP80 package but also to any other lead frame packaging technologies for RF/high-speed applications.

\section{Acknowledgments}

The research work is financially supported by the Ministry of Industry and Information Technology of China (MIIT) (Grant No. 2009ZX01031-002-002), the National Natural Science Foundation of China (No. 61040032) and the Educational Industrialization Foundation of Jiangsu Province (No. JHZD10036). 